\title{
Natalizumab for the treatment of relapsing multiple sclerosis
}

\author{
Richard A Rudick' \\ Michael A Panzara ${ }^{2}$ \\ 'Cleveland Clinic Foundation, \\ Cleveland, OH, USA; ${ }^{2}$ Biogen Idec, \\ Inc., Cambridge, MA, USA
}

Correspondence: Richard A Rudick

Cleveland Clinic Foundation, 9500 Euclid Avenue, Area UI00, Cleveland, $\mathrm{OH} 44195$, USA

Tel + I 2164451915

Fax + I 2164455192

Email rudickr@ccf.org

\begin{abstract}
Natalizumab is an $\alpha 4$-integrin antagonist approved as monotherapy for patients with relapsing multiple sclerosis (MS), based on demonstrated efficacy in the pivotal AFFIRM study $(\mathrm{N}=942)$. Natalizumab monotherapy reduced risk of disability progression by $42 \%-54 \%$ and annualized relapse rate by $68 \%$ during a period of 2 years. Natalizumab was also associated with significant reductions in number of T2-hyperintense, gadolinium-enhancing, and T1-hypointense lesions and in volume of T2-hyperintense lesions (all $\mathrm{p}<0.001$ ) on magnetic resonance imaging. Furthermore, natalizumab-treated patients in AFFIRM experienced significant improvements from baseline in the physical and mental components of the Short Form-36 ( $\mathrm{p} \leq 0.01)$ and a $35 \%$ reduction in risk of clinically significant vision loss $(p=0.008$ vs placebo). Natalizumab was well tolerated in phase 3 studies. Common adverse events were generally mild and included headache, fatigue, urinary tract infections, and arthralgia. Serious adverse events were similar between treatment groups. The incidence of serious hypersensitivity reactions associated with natalizumab was $<1 \%$. Progressive multifocal leukoencephalopathy was a rare complication of treatment, observed in 2 patients with MS who received natalizumab plus interferon $\beta$ - $1 \mathrm{a}$. The robust clinical benefits of natalizumab, including benefits on patient-reported quality of life, make it an important addition to disease-modifying therapies available to patients with relapsing MS.
\end{abstract}

Keywords: multiple sclerosis, natalizumab, $\alpha 4$-integrin antagonist

\section{Introduction}

Multiple sclerosis (MS) is a chronic, inflammatory disease of the central nervous system (CNS). The disease is characterized by focal areas of demyelination and axonal loss that may be manifested as sensory disturbances, unilateral optic neuritis, diplopia, limb weakness, clumsiness, gait ataxia, neuropsychological impairment, sexual and bladder dysfunction, pain, fatigue, and spasticity (Noseworthy et al 2000). Although the pathogenesis of MS is not completely understood, it is believed that interactions between adhesion molecules on activated leukocytes and their receptors on endothelial cells of the vessel wall promote leukocyte migration across the blood-brain barrier. Once in the CNS, leukocytes may reactivate and promote recruitment of a second wave of leukocytes into the CNS. This reaction in turn promotes an inflammatory cascade that causes demyelination and axonal loss (ffrench-Constant 1994).

Natalizumab (Tysabri ${ }^{\circledR}$; Biogen Idec, Inc., Cambridge, MA, USA and Elan Pharmaceuticals, Inc., San Francisco, CA, USA), a humanized monoclonal antibody to the $\alpha 4$-subunit of $\alpha 4 \beta 1$-integrin (expressed on the surface of all leukocytes except neutrophils), is the first selective adhesion molecule inhibitor available for the treatment of MS. By binding to $\alpha 4$-integrin, natalizumab inhibits the $\alpha 4$-mediated adhesion of leukocytes to the vascular cell adhesion molecule-1 receptor expressed on vascular endothelial cells, preventing leukocyte recruitment into the CNS and subsequent inflammatory activity. Natalizumab has also been postulated to inhibit leukocyte priming 
and lymphocyte apoptosis by inhibiting interactions between $\alpha 4$-integrin and its ligands (Rudick and Sandrock 2004).

Two randomized, double-blind, placebo-controlled studies, AFFIRM (Natalizumab Safety and Efficacy in Relapsing-Remitting Multiple Sclerosis) and SENTINEL (Safety and Efficacy of Natalizumab in Combination With Interferon Beta-1a in Patients With Relapsing-Remitting Multiple Sclerosis), were conducted to evaluate the efficacy and safety of natalizumab alone (AFFIRM) or in combination with interferon $\beta$-1a (SENTINEL) in patients with relapsing MS (Polman et al 2006; Rudick et al 2006). The primary endpoints in both studies were the rate of clinical relapses at 1 year and the cumulative probability of disability progression sustained for 12 weeks at 2 years. Predefined secondary and tertiary endpoints included the effect of natalizumab on magnetic resonance imaging (MRI) outcomes, healthrelated quality of life (HRQoL), and visual function. This article reviews the available data describing the effects of natalizumab on these clinical parameters. In addition, the safety and tolerability profile of natalizumab is discussed.

\section{Efficacy of natalizumab in pivotal studies}

The efficacy of natalizumab monotherapy was evaluated in the AFFIRM study (Polman et al 2006). Key inclusion criteria were similar to pivotal studies of disease-modifying therapies (DMTs) for MS (IFNB Study Group 1993; Jacobs et al 1996; Johnson et al 1995; PRISMS Study Group 1998) and included a diagnosis of relapsing MS according to McDonald criteria (McDonald et al 2001), Expanded Disability Status Scale (EDSS) scores from 0 to 5.0, MRI lesions consistent with MS, and at least 1 medically documented relapse within the 12 months before randomization. Patients were excluded from the study if they had progressive forms of MS (Lublin and Reingold 1996); had a relapse within 50 days of study drug administration; or were treated with interferon $\beta$, glatiramer acetate, or both for more than 6 months. Enrolled patients were randomized in a $2: 1$ ratio to receive an intravenous infusion of natalizumab $300 \mathrm{mg}$ or placebo every 4 weeks for up to 116 weeks (Polman et al 2006).

The SENTINEL study evaluated the efficacy of natalizumab added to intramuscular interferon $\beta$-1a $\left(\right.$ Avonex ${ }^{\circledR}$; Biogen Idec, Inc., Cambridge, MA, USA). In addition to the key inclusion criteria in the AFFIRM study, patients entering the SENTINEL study had received treatment with intramuscular interferon $\beta$-1a for at least 12 months before randomization and had at least 1 relapse in the prior year despite interferon therapy. Patients with progressive forms of MS (Lublin and Reingold 1996), those who experienced a relapse within 50 days before study drug administration, or those who received treatment with an approved DMT other than intramuscular interferon $\beta$-1a once weekly within the 12 months before randomization were excluded from the study. Enrolled patients were randomized to receive an intravenous infusion of natalizumab $300 \mathrm{mg}$ or placebo every 4 weeks in addition to an intramuscular injection of interferon $\beta$-1a (30 $\mu \mathrm{g}$ ) once weekly for up to 116 weeks (Rudick et al 2006).

Primary endpoints in both studies were the rate of clinical relapses at 1 year and the cumulative probability of disability progression sustained for at least 12 weeks during the 2-year study period. Relapses were defined as any new or recurrent neurologic symptoms not associated with fever or infection, lasting at least 24 hours, and accompanied by new objective neurologic findings upon examination. Disability progression was defined as at least a 1.0-point increase in EDSS score from a baseline score $\geq 1.0$ or a 1.5-point increase from a baseline score of 0 , sustained for at least 12 weeks. Secondary 1-year endpoints in both studies were the proportion of relapse-free patients, the number of new or enlarging T2-hyperintense lesions, and the number of gadolinium-enhancing $(\mathrm{Gd}+)$ lesions. Secondary 2-year endpoints in both studies were the rate of clinical relapses, disease progression as measured by the Multiple Sclerosis Functional Composite (MSFC) (Rudick et al 1997), the number of new T1-hypointense lesions, and the volume of T2-hyperintense lesions. Tertiary endpoints in both studies included the effects of natalizumab on brain atrophy, HRQoL, and visual function.

A total of 942 patients $(n=627$ natalizumab; $n=315$ placebo) were enrolled in the AFFIRM study. Baseline demographics of these patients were similar to those of patients with MS who participated in pivotal studies of DMTs (IFNB Study Group 1993; Jacobs et al 1996; Johnson et al 1996; PRISMS Study Group 1998). The mean age of patients was 36 years, and the mean number of relapses within the 12 months before randomization was 1.52. The mean EDSS score was 2.3, and the median disease duration was 5 years. Patients in the SENTINEL study had similar baseline demographics and disease characteristics. Of the 1171 patients enrolled in SENTINEL $(\mathrm{n}=589$ natalizumab + interferon $\beta$-1a; $n=582$ placebo + interferon $\beta$-1a), the mean age of patients was 39 years and the mean number of relapses within the 12 months before randomization was 1.47. Mean EDSS score was 2.4, and the median disease duration was 7 years (Polman et al 2006; Rudick et al 2006). 


\section{Clinical outcomes}

In the AFFIRM study, natalizumab monotherapy reduced the risk of disability progression sustained for 12 weeks over 2 years by $42 \%$ compared with placebo; $17 \%$ of patients treated with natalizumab experienced disease progression compared with $29 \%$ of those treated with placebo (hazard ratio $[\mathrm{HR}]=0.58 ; 95 \%$ confidence interval $[\mathrm{CI}]=0.43-0.77$; $\mathrm{p}<0.001$ ). In a sensitivity analysis, natalizumab reduced the risk of progression of disability sustained for 24 weeks over 2 years by $54 \%$ compared with placebo (HR $=0.46 ; 95 \%$ $\mathrm{CI}=0.33-0.64 ; \mathrm{p}<0.001)$ (Polman et al 2006). Moreover, natalizumab significantly reduced disability progression as measured by the MSFC compared with placebo. A significant difference between the groups in change from baseline in MSFC z-score was apparent 12 weeks after start of treatment ( $p<0.001)$, an effect that was maintained over the 2-year study (Balcer et al 2005; Phillips et al 2006).

Natalizumab monotherapy was also associated with significant improvements in relapse-related outcomes. At 1 year, natalizumab reduced the annualized relapse rate by $68 \%$ compared with placebo ( 0.26 natalizumab vs 0.81 placebo; $\mathrm{p}<0.001)$, and this reduction was maintained at 2 years $(0.23$ natalizumab vs 0.73 placebo; $\mathrm{p}<0.001)$. This result suggests that the effect of natalizumab on relapses is substantially greater than that of other MS treatments, which have been shown to reduce MS relapses over 2 years by up to $34 \%$ (IFNB Study Group 1993; Jacobs et al 1996; Johnson et al 1996; Polman et al 2006; PRISMS Study Group 1998; US FDA PCNS Advisory Committee 2006). The proportion of relapsefree patients was also significantly greater in the natalizumab group compared with the placebo group at 1 year $(77 \%$ vs $56 \% ; \mathrm{p}<0.001)$. A similar effect was observed at 2 years (67\% natalizumab vs $41 \%$ placebo; $p<0.001$ ) (Polman et al 2006), which was maintained for up to 3 years in an open-label extension study (O'Connor et al 2007).

In the SENTINEL study, natalizumab added to interferon $\beta-1$ a reduced the risk of disability progression sustained for 12 weeks over 2 years by $24 \%$ compared with interferon $\beta-1 \mathrm{a}$ alone $(\mathrm{HR}=0.76 ; 95 \% \mathrm{CI}=0.61-0.96 ; \mathrm{p}=0.02)$. In a sensitivity analysis of the risk of disability progression sustained for 24 weeks, no significant differences were found between the treatment groups ( $15 \%$ for combination therapy vs $18 \%$ for interferon $\beta$-1a alone; $\mathrm{HR}=0.82 ; 95 \% \mathrm{CI}=0.61-1.09$; $\mathrm{p}=0.17$ ). The addition of natalizumab to interferon $\beta-1 \mathrm{a}$ significantly reduced disability progression as measured by the MSFC compared with interferon $\beta$-1a alone. A significant difference between the groups in change from baseline in MSFC z-score was apparent 48 weeks after beginning treatment ( $\mathrm{p} \leq 0.005)$, and this effect was maintained over the 2-year study (Data on file, Biogen Idec, Inc. 2007).

As in AFFIRM, natalizumab was also associated with significant reductions in relapse-related outcomes in SENTINEL. The addition of natalizumab to interferon $\beta$ - 1 a resulted in a 54\% reduction in annualized relapse rate at 1 year $(0.38$ for interferon $\beta-1 \mathrm{a}+$ natalizumab vs 0.82 interferon $\beta$-1a alone; $\mathrm{p}<0.001$ ); at 2 years, the relative reduction was $55 \%$ ( 0.34 for interferon $\beta-1 \mathrm{a}+$ natalizumab vs 0.75 interferon $\beta$-1a alone; $p<0.001)$. Furthermore, the proportion of relapse-free patients at 2 years was $54 \%$ in the combination therapy group compared with $32 \%$ in the interferon $\beta-1$ a alone group ( $\mathrm{p}<0.001$ ) (Rudick et al 2006).

\section{MRI outcomes}

In AFFIRM, natalizumab monotherapy caused marked reductions in the number and volume of MRI lesions. At 1 year, the mean number of new or enlarging T2-hyperintense lesions was 1.2 in natalizumab-treated patients compared with 6.1 in placebo-treated patients $(\mathrm{p}<0.001)$. Furthermore, natalizumab reduced the mean number of new or enlarging T2-hyperintense lesions by $83 \%$ over 2 years compared with placebo (1.9 vs 11.0; $\mathrm{p}<0.001$ ). Natalizumab also reduced the mean number of $\mathrm{Gd}+$ lesions by $92 \%$ at 1 and 2 years compared with placebo $(p<0.001)$ (Polman et al 2006) and the mean number of new T1-hypointense lesions by $76 \%$ over 2 years compared with placebo (1.1 vs 4.6; $p<0.001$ ) (Miller et al 2007). Median T2-hyperintense lesion volume was significantly lower in natalizumab-treated patients compared with placebo-treated patients at 2 years $\left(9175 \mathrm{~mm}^{3} \mathrm{vs}\right.$ $11,137 \mathrm{~mm}^{3} ; \mathrm{p}<0.001$ ) (Miller et al 2007).

Brain atrophy can be reliably assessed in patients with MS by measuring the brain parenchymal fraction (BPF), which is the ratio of brain parenchymal volume to the total volume within a smoothed surface contour around the brain (Rudick et al 1999). During 2 years of treatment in AFFIRM, the change from baseline BPF was similar in natalizumaband placebo-treated patients (mean percent change in $\mathrm{BPF}=-0.80 \% \mathrm{vs}-0.82 \% ; \mathrm{p}=0.822$ ). Although both groups exhibited a decrease in BPF from baseline to 1 year, a relatively greater reduction was observed in the natalizumab group compared with the placebo group $(-0.56 \%$ vs $-0.40 \%$; $\mathrm{p}=0.002$ ). In the second year, the mean percent change in BPF was significantly less in the natalizumab group compared with the placebo group $(-0.24 \%$ vs $-0.43 \%$; $p=0.004)$, indicating a significant slowing of atrophy compared with placebo (Miller et al 2007). It is speculated that the greater decrease in BPF observed in natalizumab-treated patients 
during the first year of treatment may have been attributable to a reduction in inflammation and edema, which can cause an acute shrinkage of brain tissue known as pseudoatrophy (Fisher et al 2006).

In the SENTINEL study, the addition of natalizumab to interferon $\beta$-1a resulted in significant reductions in the number of MRI lesions. At 1 year, the mean number of new or enlarging T2-hyperintense lesions was 0.5 in patients treated with natalizumab plus interferon $\beta$-1a compared with 2.4 in patients treated with interferon $\beta$-1a alone $(\mathrm{p}<0.001)$. Furthermore, the mean number of new or enlarging T2-hyperintense lesions was reduced by $83 \%$ over 2 years ( 0.9 interferon $\beta$-1a + natalizumab vs 5.4 interferon $\beta$-1a alone; $p<0.001)$. In addition, natalizumab added to interferon $\beta$-1a reduced the mean number of $\mathrm{Gd}+$ lesions by $88 \%$ at 1 year $(0.1$ interferon $\beta$-1a + natalizumab vs 0.8 interferon $\beta$-1a alone; $\mathrm{p}<0.001)$ and by $89 \%$ at 2 years ( 0.1 interferon $\beta$-1a + natalizumab vs 0.9 interferon $\beta$-1a alone; $p<0.001)$ compared with interferon $\beta$-1a alone (Rudick et al 2006). The mean number of new T1-hypointense lesions was reduced by $44 \%$ over 2 years (2.3 interferon $\beta$-1a + natalizumab vs 4.1 interferon $\beta$-1a alone; $p<0.001)$. Median T2-hyperintense lesion volume was significantly lower in patients receiving interferon $\beta$-1a plus natalizumab compared with patients receiving interferon $\beta$-1a alone at 2 years $\left(5848 \mathrm{~mm}^{3}\right.$ vs $\left.7260 \mathrm{~mm}^{3} ; \mathrm{p}<0.001\right)$ (Data on file, Biogen Idec, Inc. 2007).

Measurements of changes in BPF at 1 and 2 years in the SENTINEL study revealed patterns of reduction in the interferon $\beta$-1a plus natalizumab and interferon $\beta$-1a alone treatment groups that were similar to those observed in the placebo and natalizumab monotherapy groups, respectively, in AFFIRM. During 2 years of treatment in SENTINEL, the changes in BPF from baseline were similar in the natalizumab plus interferon $\beta$-1a and interferon $\beta$-1a alone treatment groups $(-0.81 \%$ vs $-0.82 \%, p=0.926)$. In the first year, however, there was a greater reduction in BPF in the natalizumab plus interferon $\beta$-1a group $(-0.5 \%$ vs $-0.42 \%$, $\mathrm{p}=0.058)$, whereas the reduction in the second year was greater in the interferon $\beta$ - 1 a alone group $(-0.40 \%$ vs $-0.31 \%$, $\mathrm{p}=0.020)$ (Data on file, Biogen Idec, Inc. 2007).

\section{Clinical and MRI efficacy in AFFIRM}

Post hoc analyses were conducted in patients from the AFFIRM study to determine the ability of natalizumab to achieve a complete clinical and MRI response. Absence of clinical disease activity was defined as no relapses and no disability progression, sustained for 12 weeks. Absence of MRI disease activity was defined as no Gd+ lesions at 1 and
2 years and no new or enlarging T2-hyperintense lesions during the 2 years. The proportions of patients who were free of clinical activity, MRI activity, and a more stringent definition of clinical and MRI disease activity over 2 years were determined. Significantly more natalizumab-treated patients were free of clinical disease activity $(64.3 \%$ vs $38.9 \%$; $\mathrm{p}<0.0001)$, MRI disease activity ( $57.7 \%$ vs $14.2 \%$; $\mathrm{p}<0.0001)$, and both clinical and MRI disease activity $(36.7 \%$ vs $7.2 \%$; $<0.0001$ ) compared with placebo-treated patients over the 2 years of the study (Havrdova et al 2007).

\section{Clinical and MRI efficacy in patients with highly active MS}

Post hoc analyses of patients with highly active relapsing MS ( $\geq 2$ relapses in the year prior to study entry and $\geq 1 \mathrm{Gd}+$ lesion on T1-weighted MRI at study entry) were performed for AFFIRM and SENTINEL. In AFFIRM patients with highly active disease ( $\mathrm{n}=148$ natalizumab; $\mathrm{n}=61$ placebo), natalizumab monotherapy reduced the risk of disability progression sustained for 12 weeks by $53 \%$ ( $\mathrm{HR}=0.47 ; 95 \%$ $\mathrm{CI}=0.24-0.93 ; \mathrm{p}=0.029)$ and progression sustained for 24 weeks by $64 \%(\mathrm{HR}=0.36 ; 95 \% \mathrm{CI}=0.17-0.76 ; \mathrm{p}=0.008)$ compared with placebo. In addition, natalizumab reduced the 2 -year annualized relapse rate by $81 \%$ compared with placebo ( 0.28 vs 1.46 , respectively; $p<0.001)$ in these patients. Furthermore, significant improvements on all MRI outcomes were observed with natalizumab compared with placebo in patients with highly active disease (Data on file, Biogen Idec, Inc. 2007; European Union TYSABRI SmPC 2006).

In SENTINEL patients with highly active disease $(n=74$ interferon $\beta$ - $1 \mathrm{a}+$ natalizumab; $\mathrm{n}=95$ interferon $\beta$-1a alone), the addition of natalizumab to interferon $\beta$-1a reduced the risk of disability progression sustained for 12 weeks by $61 \%$ $(\mathrm{HR}=0.39 ; 95 \% \mathrm{CI}=0.21,0.74 ; \mathrm{p}=0.004)$ and sustained for 24 weeks by $58 \%(\mathrm{HR}=0.42, \mathrm{CI}=0.19,0.95 ; \mathrm{p}=0.038)$ compared with interferon $\beta$-1a alone. The addition of natalizumab to interferon $\beta$-1a also led to a $76 \%$ reduction in the 2-year annualized relapse rate compared with interferon $\beta$-1a alone $(0.28$ vs $1.16 ; p<0.001)$. Furthermore, significant improvements on all MRI outcomes were observed in the interferon $\beta$-1a plus natalizumab group compared with the interferon $\beta$-1a group in highly active patients (Data on file, Biogen Idec, Inc. 2007).

\section{HRQoL}

The chronic symptoms of MS interfere with activities of daily living, resulting in significantly reduced HRQoL. In contrast to the clinical and MRI outcomes that are measured by physicians, 
HRQoL endpoints provide an assessment of a therapy on domains regarded by patients as key factors in determining their overall health status. The majority of phase 3 studies in patients with MS lack HRQoL measures; thus, a rigorous assessment of HRQoL was performed in AFFIRM and SENTINEL. HRQoL was evaluated using the Short Form-36 (SF-36) (Ware et al 1992) and the visual analog scale (VAS), a subjective global assessment of well-being. To date, the SF-36 has not been used to assess HRQoL in pivotal trials of other MS treatments. In AFFRIM and SENTINEL it was administered as a part of the Multiple Sclerosis Quality of Life Inventory (Consortium of Multiple Sclerosis Centers 1997). Two summary scores from the SF-36 were calculated: the Physical Component Summary (PCS) and the Mental Component Summary (MCS). The SF36 and the VAS were administered to patients enrolled in both AFFIRM and SENTINEL at baseline and at weeks 24, 52, and 104 (Rudick et al 2007).

Data from AFFIRM and SENTINEL $(\mathrm{N}=2113)$ were combined to evaluate the burden of MS on HRQoL. The baseline mean PCS and MCS scores were $43.2 \pm 0.4$ and $47.0 \pm 0.5$, respectively, and were significantly lower than the mean scores for the general US population (50.0) (Rudick et al 2007). Furthermore, at study entry, the PCS scores for patients with an EDSS score $\geq 2.0$ were significantly lower than for those patients with an EDSS score of 0 ( $p<0.005)$ (Rudick et al 2007). Similarly, VAS scores for patients with EDSS scores $\geq 1.5$ were significantly lower compared with those for patients with an EDSS score of 0 ( $p<0.05)$. Taken together, these data provide evidence that HRQoL is significantly reduced in patients with relapsing MS.

In AFFIRM, natalizumab monotherapy was associated with significant improvements in PCS $(p=0.003)$ and MCS

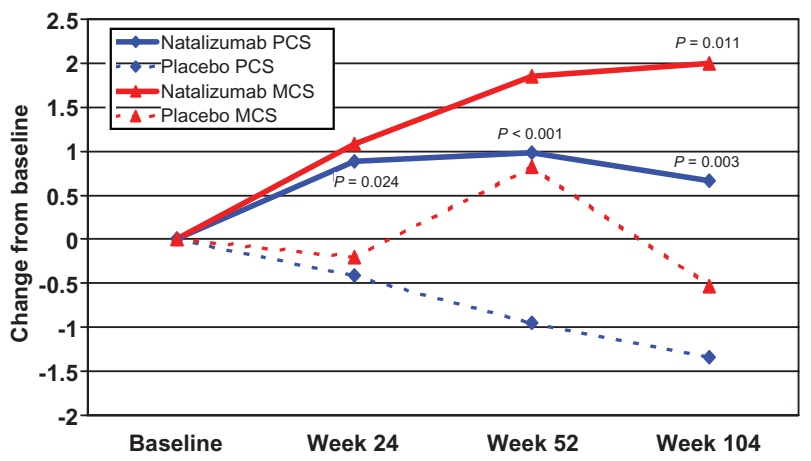

Figure I Mean changes from baseline in Physical Component Summary (PCS) and Mental Component Summary (MCS) scores of the Short Form-36 in patients from the AFFIRM study. Reprinted from Rudick RA, Miller D, Hass S, et al. 2007. Healthrelated quality of life in multiple sclerosis: effects of natalizumab. Ann Neurol, 62:335-46. Copyright @ 2007 (American Neurological Association), with permission of John Wiley \& sons, Inc.
( $p=0.011)$ scores at week 104 (Figure 1). In fact, changes from baseline in PCS scores were significantly higher at weeks 24, 52, and 104 compared with placebo. In SENTINEL, changes from baseline in PCS scores for patients treated with interferon $\beta$-1a plus natalizumab were significantly higher at weeks $52(\mathrm{p}=0.014)$ and $104(\mathrm{p}<0.001)$ compared with scores for patients treated with interferon $\beta$-1a alone (Rudick et al 2007). Additionally, a greater percentage of natalizumabtreated patients improved and a lower percentage worsened by a clinically important amount (defined as a $\geq 0.5$ standard deviation change from baseline to week 104 in PCS and MCS scores) compared with placebo- or interferon $\beta$-1a-treated patients in both studies (Rudick et al 2007). Findings from the subject global assessment of well-being were similar to those from the SF-36. Changes from baseline in VAS scores for patients treated with natalizumab monotherapy were greater than those for patients treated with placebo at weeks $52(\mathrm{p}=$ 0.042 ) and $104(\mathrm{p}=0.007)$ (Rudick et al 2007).

\section{Visual function}

Visual function is an important measure of neurologic impairment in patients with relapsing MS. For example, remitting episodes of acute optic neuritis can influence the overall visual function of a patient. Thus, prespecified analyses were performed in AFFIRM and SENTINEL to determine the effect of natalizumab on visual function (Balcer et al 2007). Visual acuity was measured using the Sloan letter chart at 3 contrast levels: 100\% (high-contrast), 2.5\% (low-contrast), and $1.25 \%$ (low-contrast). Visual function testing was performed every 12 weeks using a standardized protocol based on visual acuity testing methods from the Early Treatment Diabetic Retinopathy Study (Balcer et al 2007). Sloan chart scores for each contrast level were calculated based on the number of letters correctly identified by the patient. The cumulative probability of clinically significant worsening of visual function, defined as 2-line (10-letter) reductions in Sloan chart scores persisting over 12 weeks, was determined using Kaplan-Meier analysis (Balcer et al 2007).

When low-contrast Sloan charts were used, patients treated with natalizumab had lower cumulative probabilities of sustained clinically significant worsening of visual function compared with placebo or interferon $\beta$-1a alone. In AFFIRM, natalizumab reduced the risk of sustained clinically significant worsening of visual function by $47 \%(\mathrm{HR}=0.53$; $95 \% \mathrm{CI}=0.36-0.76 ; \mathrm{p}<0.001)$ at the $2.5 \%$ contrast level and by $35 \%(\mathrm{HR}=0.65 ; 95 \% \mathrm{CI}=0.47-0.90 ; \mathrm{p}=0.008)$ at the $1.25 \%$ contrast level compared with placebo (Figure 2) (Balcer et al 2007). 


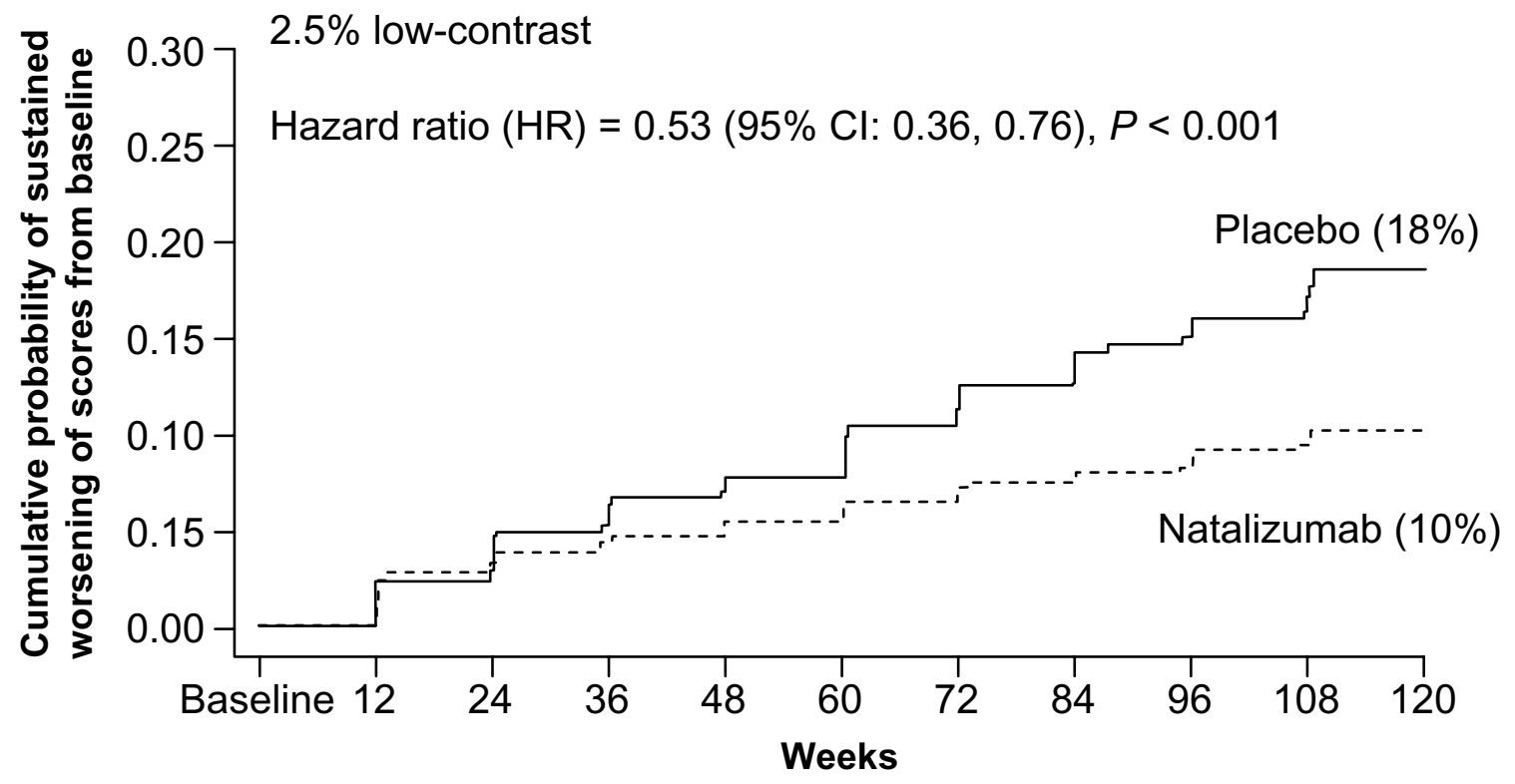

No. at risk

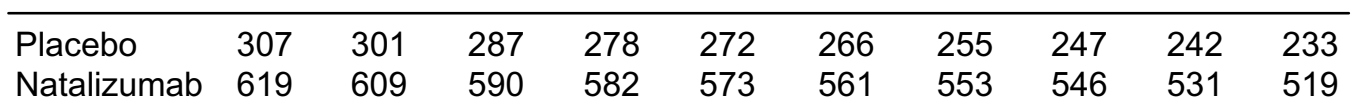

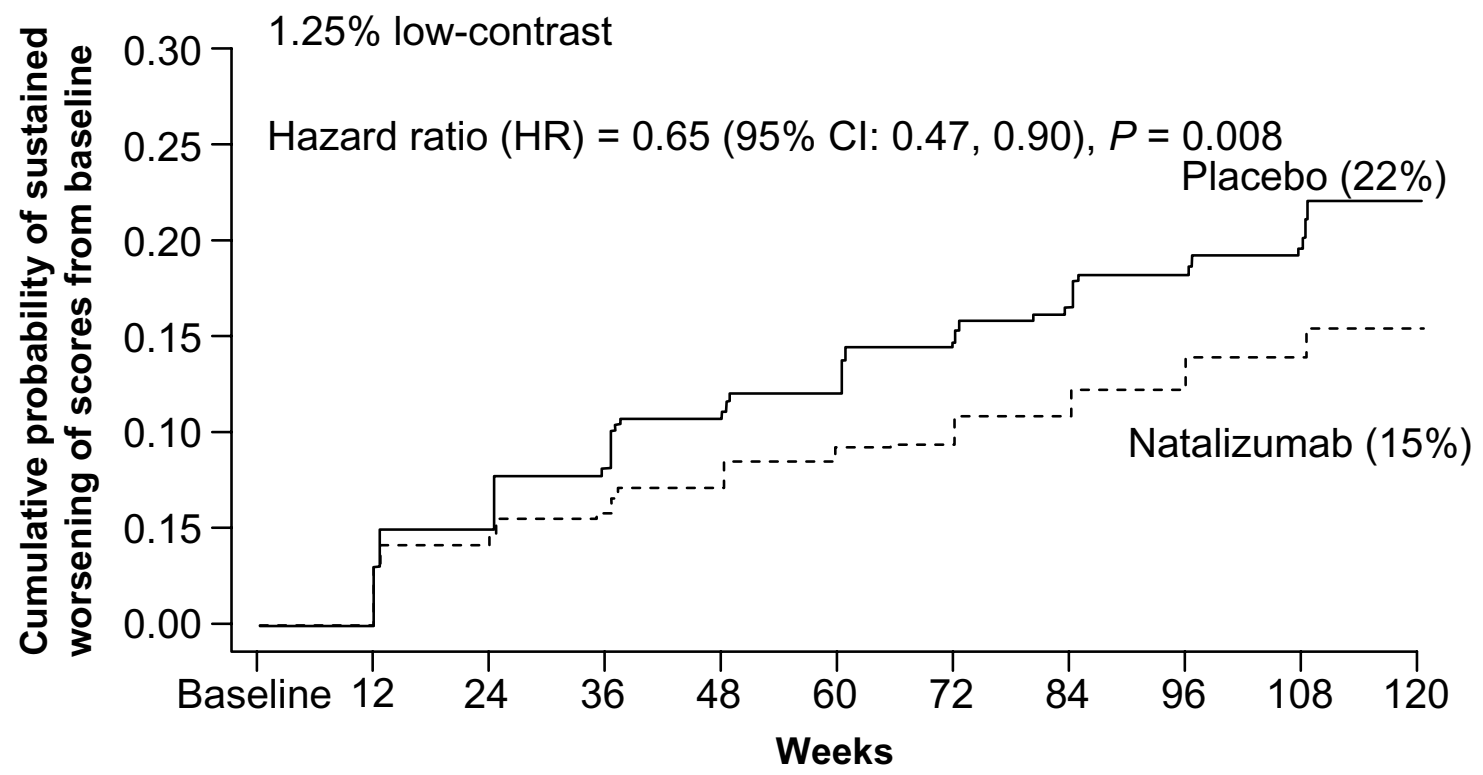

No. at risk

\begin{tabular}{lcccccccccc}
\hline Placebo & 307 & 296 & 278 & 267 & 261 & 254 & 245 & 235 & 232 & 222 \\
Natalizumab & 619 & 598 & 580 & 569 & 556 & 544 & 534 & 520 & 504 & 491
\end{tabular}

Figure 2 Kaplan-Meier plots of the time to sustained worsening of vision scores from baseline during the AFFIRM study. The cumulative probability of sustained worsening of visual function was defined as 2-line (I0-letter) reductions in Sloan chart scores persisting over I2 weeks. Reprinted from Balcer LJ, Galetta SL, Calabresi PA, et al. 2007. Natalizumab reduces visual loss in patients with relapsing multiple sclerosis. Neurology, 68:1299-304. Copyright (C) 2007, with permission from Lippincott, Williams \& Wilkins. 
In SENTINEL, the cumulative probability of sustained clinically significant worsening of visual function was lower for both low-contrast Sloan charts in patients treated with interferon $\beta$-1a plus natalizumab compared with patients treated with interferon $\beta$-1a alone. However, clinically significant differences were demonstrated only at the $1.25 \%$ contrast level $(\mathrm{HR}=0.72 ; 95 \% \mathrm{CI}=0.54-0.98 ; \mathrm{p}=0.038)$. In addition, differences between treatment groups occurred later over the 2 -year treatment period. These findings were attributed to the use of an active-comparator treatment arm, which may require longer follow-up periods and more sensitive treatment measures to detect treatment effects (Balcer et al 2007).

\section{Immunogenicity of natalizumab}

Antibody formation is an expected response to treatment with therapeutic proteins, and the formation of such antibodies has been demonstrated to reduce the efficacy of the product (Schellekens and Casadevall 2004). In the AFFIRM and SENTINEL studies, patients were tested for the development of anti-natalizumab antibodies every 12 weeks throughout the study. Serum concentrations of anti-natalizumab antibodies were determined by an enzyme-linked immunosorbent assay, and patients were defined as being antibody negative $(<0.5 \mu \mathrm{g} / \mathrm{mL}$ at all time points $)$, transiently positive ( $\geq 0.5 \mu \mathrm{g} / \mathrm{mL}$ at a single time point), or persistently positive ( $\geq 0.5 \mu \mathrm{g} / \mathrm{mL}$ at 2 or more time points at least 6 weeks apart) (Calabresi et al 2007).

In AFFIRM, 57 natalizumab patients (9\%) developed anti-natalizumab antibodies; $3 \%(n=20)$ were transiently positive, and $6 \%(\mathrm{n}=37)$ were persistently positive. During SENTINEL, $12 \%$ of patients $(n=70)$ developed anti-natalizumab antibodies; $5 \%(\mathrm{n}=32)$ were transiently positive, and $6 \%(\mathrm{n}=38)$ were persistently positive (Calabresi et al 2007). Most patients who developed anti-natalizumab antibodies did so within 12 weeks of starting natalizumab treatment (88\% AFFIRM; 96\% SENTINEL) (Calabresi et al 2007). Notably, the presence of anti-natalizumab antibodies was associated with a reduction in trough serum natalizumab concentrations (Calabresi et al 2007). Persistent antibodies were associated with a loss of efficacy as measured by clinical and MRI outcomes. In contrast, transiently positive patients experienced delayed therapeutic effects of natalizumab, but full efficacy was eventually achieved with continued treatment (Calabresi et al 2007).

\section{Tolerability and safety}

Overall, natalizumab was well tolerated in the AFFIRM and SENTINEL studies. In AFFIRM, infusion reactions, defined as any event occurring within 2 hours of the start of infusion, occurred in $24 \%$ of natalizumab-treated patients compared with $18 \%$ of placebo patients $(\mathrm{p}=0.04)$ (Polman et al 2006). Similarly, in SENTINEL, the incidence of infusion reactions was $24 \%$ in the interferon $\beta$-1a plus natalizumab group and $20 \%$ in the interferon $\beta-1$ a alone group $(\mathrm{p}=0.11)$. The most common of these reactions was headache, which did not usually result in discontinuation of natalizumab treatment. Hypersensitivity reactions were defined as reports of hypersensitivity, allergic reaction, or anaphylactic/anaphylactoid reactions and any reports of urticaria, allergic dermatitis, or hives. In AFFIRM, the incidence of hypersensitivity reactions associated with natalizumab was $4 \%$ and the incidence of anaphylactic/anaphylactoid reactions was $0.8 \%$ (Polman et al 2006). In SENTINEL, 11 patients (1.9\%) receiving combination therapy experienced a hypersensitivity reaction, and there were no reports of anaphylactic or anaphylactoid reactions associated with natalizumab (Rudick et al 2006). An evaluation of hypersensitivity reactions according to anti-natalizumab antibody status revealed that $46 \%$ of 37 persistently positive patients and $15 \%$ of 20 transiently positive patients in AFFIRM experienced a hypersensitivity reaction. The corresponding values for patients in SENTINEL were $21 \%$ of 38 persistently positive patients and none of 32 transiently positive patients (Calabresi et al 2007). Patients who experienced hypersensitivity reactions while receiving natalizumab during the studies discontinued treatment.

As previously stated, non-hypersensitivity-mediated infusion reactions did not necessitate discontinuation of natalizumab therapy in AFFIRM or SENTINEL. However, infusion reactions that occurred over multiple natalizumab infusions were associated with persistent positivity for anti-natalizumab antibodies. Therefore, testing for antinatalizumab antibodies should be considered in the setting of recurrent infusion-related symptoms. Two consecutive positive blood samples separated by $2-3$ months after at least 6 months' treatment confirm persistent antibody-positive status. Discontinuation of natalizumab should be strongly considered once persistent positivity is confirmed.

Generally, few differences in adverse event profiles were observed between natalizumab and placebo or interferon $\beta$-1a groups in AFFIRM and SENTINEL. Common adverse events were generally mild and included headache, fatigue, urinary tract infections, and arthralgia (Tysabri ${ }^{\circledR}$ package insert 2007). In AFFIRM, patients treated with natalizumab monotherapy experienced more fatigue $(27 \%$ vs $21 \%$; $=0.048)$ and allergic reactions ( $9 \%$ vs $4 \% ; \mathrm{p}=0.012$ ) compared with those who received placebo (Polman et al 2006). During the 
SENTINEL study, patients treated with natalizumab plus interferon $\beta$-1a experienced significantly more pharyngitis ( $7 \%$ vs $4 \% ; \mathrm{p} \leq 0.05)$, anxiety $(12 \%$ vs $8 \% ; \mathrm{p} \leq 0.01)$, sinus congestion ( $6 \%$ vs $3 \% ; p \leq 0.01)$, and peripheral edema $(5 \%$ vs $1 \% ; p \leq 0.001)$ compared with patients who received interferon $\beta$-1a alone (Rudick et al 2006). In both studies, the incidence of infection was similar between the treatment groups (AFFIRM, 79\% in natalizumab and placebo groups [Figure 3]; SENTINEL, $83 \%$ in the interferon $\beta$ - $1 \mathrm{a}+$ natalizumab group vs $81 \%$ in interferon $\beta$-1a alone) (Polman et al 2006; Rudick et al 2006).

Laboratory testing has shown that natalizumab causes reversible elevations in circulating lymphocytes, monocytes, eosinophils, basophils and nucleated red blood cells (but not neutrophils). Moreover, rare cases of clinically significant hepatoxicity have been reported in the postmarketing setting (Tysabri ${ }^{\circledR}$ package insert 2007). Regular laboratory monitoring during natalizumab treatment is not considered necessary.

In February 2005, commercial and clinical trial dosing of natalizumab was voluntarily suspended after 2 reports of progressive multifocal leukoencephalopathy (PML), a serious, sometimes fatal, demyelinating disease of the CNS that results from reactivation of the latent JC virus and that usually occurs in immunocompromised patients (Major et al 1992). Both patients had received natalizumab plus interferon $\beta$-1a in SENTINEL (Kleinschmidt-DeMasters and Tyler 2005; Langer-Gould et al 2005). A third case of PML was retrospectively identified in a patient who was treated with natalizumab for Crohn's disease in a clinical study (Van Assche et al 2005). Subsequent to these reports of PML, an extensive safety evaluation revealed no additional cases of PML among 3116 patients who received natalizumab in clinical studies.

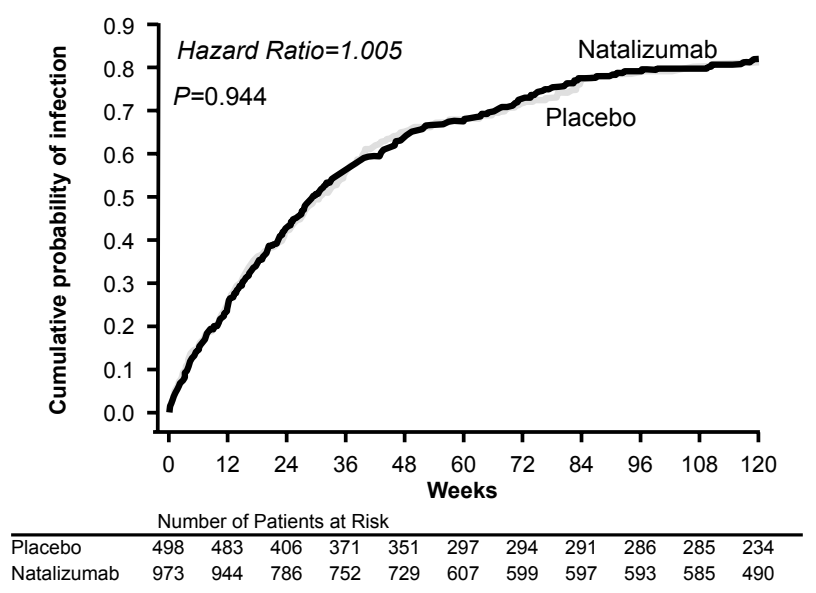

Figure 3 Cumulative probability of infection in the AFFIRM study (unpublished data on file, Biogen Idec).
The risk of developing PML associated with natalizumab use was determined to be 1 in 1000 patients $(95 \% \mathrm{CI}=0.2,2.8$ in 1000 patients) over 17.9 months of treatment (Yousry et al 2006). Natalizumab was reintroduced in the United States and approved in the European Union in June 2006.

To better assess the safety profile of natalizumab, a comprehensive risk management plan that includes the Tysabri ${ }^{\circledR}$ Outreach: Unified Commitment to Health ( $\mathrm{TOUCH}^{\mathrm{TM}}$ ) Prescribing Program and the Tysabri ${ }^{\circledR}$ Global Observation Program in Safety (TYGRIS) observational cohort study was developed. The TOUCH ${ }^{\mathrm{TM}}$ Prescribing Program is a mandatory prescribing program in the United States. All physicians, infusion sites, and patients are required to enroll before natalizumab treatment is initiated, and reauthorization is required every 6 months. The objectives of TOUCH ${ }^{\mathrm{TM}}$ are to ensure appropriate and informed use of natalizumab, assess the incidence of serious opportunistic infections, including PML, in patients treated with natalizumab, and monitor natalizumab-treated patients for signs and symptoms of PML. TYGRIS is a voluntary global observational study designed to investigate the long-term safety of natalizumab in the clinical practice setting. A recent update of safety data collected from the TOUCH ${ }^{\mathrm{TM}}$ and TYGRIS programs showed that the safety profile of natalizumab was consistent with that seen in earlier clinical studies (Panzara et al 2007). Global utilization data released in January, 2008, indicate that approximately 30,900 patients have been treated with natalizumab in the combined clinical trial and postmarketing settings, and of these patients, approximately 6,300 have received natalizumab for at least 1 year (unpublished data on file, Biogen Idec). In addition to TOUCH ${ }^{\mathrm{TM}}$ and TYGRIS, the Tysabri ${ }^{\circledR}$ Observational Program in Europe will provide further insight into the long-term effects of natalizumab. Currently, several initiatives to better understand PML and its management in the context of natalizumab therapy are being planned or conducted.

\section{Patient selection and PML diagnostic algorithm}

A recent publication by a panel of experts in neurology, neuroradiology, and PML provides guidelines to help with the selection of appropriate candidates for natalizumab therapy and with the diagnosis of suspected PML (Kappos et al 2007). The article recommends that patients who have received prior treatment with immunosuppressants or antineoplastic agents should be carefully assessed for ongoing immunosuppression before initiating natalizumab. Recommendations based on clinical and laboratory markers to help identify 
immunocompetence in patients who have been exposed to immunosuppressive therapy have been published separately by Gold and colleagues (Gold et al 2007). A pretreatment cranial MRI scan is also recommended for all patients within 3 months of commencing natalizumab therapy to help in the assessment of subsequent new or worsening neurologic symptoms that may develop during treatment.

It is further recommended in the article that new or worsening neurological symptoms should lead to immediate suspension of natalizumab dosing and completion of a thorough neurologic assessment (Figure 4). Continued suspicion of PML after the neurologic assessment warrants a cranial MRI scan with gadolinium enhancement for comparison with the baseline pretreatment MRI scan and, if PML is still a concern, cerebrospinal fluid (CSF) testing for JC virus (JCV) DNA is recommended. However, CSF is usually negative for JCV DNA in the early stages of PML. Therefore, if the disease is still suspected after a negative CSF test, diagnostic work up should continue and natalizumab treatment should not be restarted. Clinical and MRI characteristics that may help to distinguish between PML and MS are presented in Table 1 (Kappos et al 2007).

At present, there is no proven treatment for PML. However, experience with PML in organ transplant recipients and patients with HIV strongly suggests that reconstitution of the immune system is associated with a better prognosis (Clifford et al 1999; Crowder et al 2005; Shitrit et al 2005). A recent study by Khatri et al provided preliminary evidence that accelerated removal of natalizumab from circulation via plasma exchange may be a method to reconstitute the immune system in natalizumab-treated patients who develop PML (Khatri et al 2007). However, because plasma exchange has not been specifically tested in natalizumab-treated patients with PML, the true efficacy of the procedure remains unknown.

\section{Prescribing considerations}

Natalizumab is indicated as monotherapy for the treatment of relapsing MS and is generally recommended for patients who have had an inadequate response to, or are unable to tolerate, alternate MS therapies. However, prescribing information in the United States does not preclude the use of natalizumab as a first-line agent for the treatment of relapsing MS (Tysabri ${ }^{\circledR}$ package insert 2007). In the European Union, natalizumab is approved as a first-line agent for patients with highly active disease ( $\geq 2$ disabling relapses in the previous year and $\geq 1 \mathrm{Gd}+$ lesion on MRI) and as a second-line therapy in patients who have experienced $\geq 1$ relapse in the previous year and $\geq 9$ T2 lesions or $\geq 1 \mathrm{Gd}+$ lesion while receiving interferon $\beta$ (European Agency for the Evaluation of Medicinal Products 2006).

\section{Summary}

Natalizumab is the first $\alpha 4$-integrin receptor antagonist available for the treatment of relapsing MS. Through its proposed mechanism of action, natalizumab provides evidence for

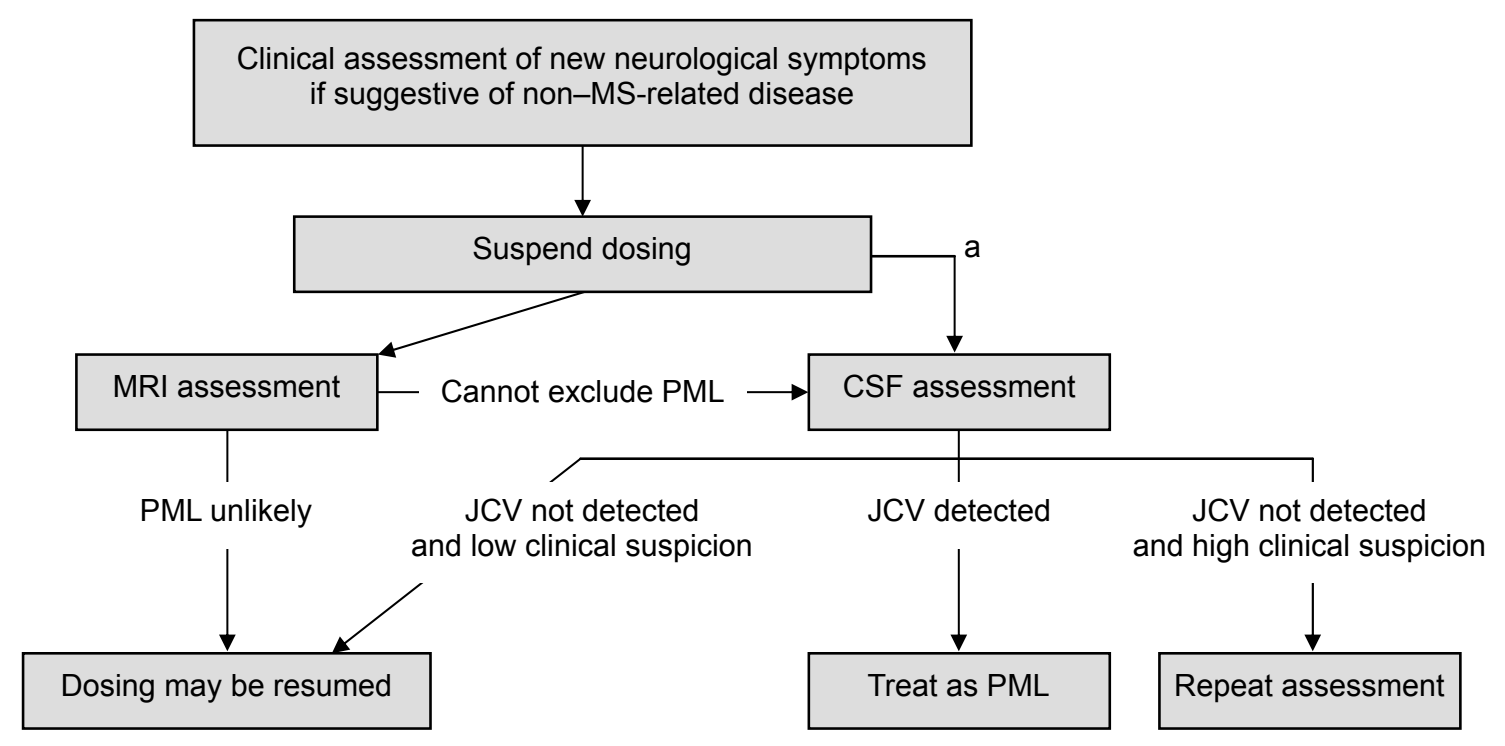

Figure 4 Diagnostic algorithm for patients with suspected progressive multifocal leukoencephalopathy (PML). Reprinted from Kappos L, Bates D, Hartung H-P, et al. 2007. Natalizumab treatment for multiple sclerosis: recommendations for patient selection and monitoring. Lancet Neurol, 6:43I-4I. Copyright @ 2007, with permission from Elsevier. alf PML is suspected on the basis of clinical presentation and MRI is not readily available, CSF assessment to exclude PML should be considered before MRI.

Abbreviations: CSF, cerebrospinal fluid; JCV, polyomavirus JC virus; MRI, magnetic resonance imaging; MS, multiple sclerosis; PML, progressive multifocal leukoencephalopathy. 
Table I Clinical and MRI characteristics of MS and PML

\begin{tabular}{|c|c|c|}
\hline & MS & PML \\
\hline \multicolumn{3}{|l|}{ Clinical characteristics $^{\mathrm{a}}$} \\
\hline Onset & Acute & Subacute \\
\hline \multirow[t]{3}{*}{ Evolution } & Hours to days & Weeks \\
\hline & Normally stabilizes & Progressive \\
\hline & Resolves spontaneously or with treatment & \\
\hline \multirow[t]{4}{*}{ Clinical presentation } & Diplopia & Cortical signs and symptoms \\
\hline & Optic neuritis & Behavioral and neuropsychological alterations \\
\hline & Incomplete myelopathy or partial myelitis & Retrochiasmal visual deficits \\
\hline & & Hemiparesis \\
\hline \multicolumn{3}{|l|}{ MRI characteristics } \\
\hline \multirow[t]{4}{*}{ Location } & Mostly focal & Diffuse, mainly subcortical \\
\hline & Widespread in brain and spinal cord & White matter tracts, sparing cortex \\
\hline & Periventricular & \\
\hline & Posterior fossa lesions often seen early & \\
\hline \multirow[t]{3}{*}{ Borders } & Sharp edges, round or finger-like & III-defined, irregular shape, \\
\hline & Confluent with other lesions & infiltrating \\
\hline & U-fibers may be involved & U-fibers destroyed \\
\hline \multirow[t]{3}{*}{ Mode of extension } & Lesions initially focal & Diffuse, asymmetric, homogenous lesions \\
\hline & Enlarge within days or weeks & No confluence with other lesions \\
\hline & Eventually decrease in size over months & Continuous progression \\
\hline Mass effect & May be seen in acute lesions & Rare, even in large lesions \\
\hline
\end{tabular}

Reprinted from Kappos L, Bates D, Hartung H-P, et al. 2007. Natalizumab treatment for multiple sclerosis: recommendations for patient selection and monitoring. Lancet Neurol, 6:43I-4I. Copyright (C) 2007, with permission from Elsevier.

aPertaining to relapse in MS.

Abbreviations: MRI, magnetic resonance imaging; MS, multiple sclerosis; PML, progressive multifocal leukoencephalopathy.

the role of circulating mononuclear leukocytes in disease pathogenesis. Natalizumab has demonstrated significant clinical and MRI efficacy in patients with relapsing MS. Natalizumab monotherapy was associated with significant reductions in annualized relapse rate, the risk of sustained disability progression, and the number and volume of MRI lesions. Additional data have shown that natalizumab reduces the risk of developing sustained visual loss and is associated with improvements in HRQoL. Natalizumab demonstrated a favorable tolerability profile in phase 3 studies; a low incidence of persistent anti-natalizumab antibody development and hypersensitivity reactions was observed, and most natalizumab-associated adverse events were generally mild. The risk of PML with natalizumab use is small, but appropriate patient selection requires an individual assessment of benefit-risk and careful monitoring.

With its demonstrated efficacy on a number of physician- and patient-measured outcomes, natalizumab represents a major advance in the treatment of relapsing MS. The available data support a favorable benefit-risk profile of natalizumab. Ongoing research and clinical experience will expand current knowledge regarding long-term efficacy and safety.

\section{Acknowledgments}

The authors thank Hema Gowda, Paul Benfield, and Matthew Hasson, Scientific Connexions, for editorial assistance in preparing this manuscript for submission.

\section{Disclosures}

Dr. Rudick has received research funding from the NIH, the National Multiple Sclerosis Society, and Biogen Idec, and consulting fees from Biogen Idec, Millenium Pharmaceuticals, Novartis, and Wyeth.

\section{References}

Balcer LJ, Galetta SL, Calabresi PA, et al. 2007. Natalizumab reduces visual loss in patients with relapsing multiple sclerosis. Neurology, 68:1299-304.

Balcer LJ, O'Connor PW, Havrdova E. 2005. The effects of natalizumab on disability progression as measured by the Multiple Sclerosis Functional Composite (MSFC) and visual function in patients with relapsing MS. Presented at 18th World Congress of Neurology; November 9; Sydney, Australia. 
Calabresi PA, Giovannoni G, Confavreux C, et al. 2007. The incidence and significance of anti-natalizumab antibodies. Neurology, 69:1391-403.

Clifford DB, Yiannoutsos C, Glickman M, et al. 1999. HAART improves prognosis in HIV-associated progressive multifocal leukoencephalopathy. Neurology, 52:623-5.

Consortium of Multiple Sclerosis Centers Health Services Research Subcommittee. 1997. Multiple Sclerosis Quality of Life Inventory: A User's Manual. New York: National Multiple Sclerosis Society.

Crowder CD, Gyure KA, Drachenberg CB, et al. 2005. Successful outcome of progressive multifocal leukoencephalopathy in a renal transplant patient. Am J Transplant, 5:1151-8.

Data on file. 2007. Cambridge, MA: Biogen Idec, Inc.

European Agency for the Evaluation of Medicinal Products. 2006.Tysabri ${ }^{\circledR}$ summary of product characteristics.

ffrench-Constant C. 1994. Pathogenesis of multiple sclerosis. Lancet, 343:271-5.

Fisher E, O'Connor PW, Havrdova E. 2006. The effects of natalizumab on brain atrophy and cognitive function: results from the AFFIRM study [abstract]. Mult Scler, 12(suppl 1):S103. Abstract P383.

Gold R, Jawad A, Miller DH, et al. 2007. Expert opinion: guidelines for the use of natalizumab in multiple sclerosis patients previously treated with immunomodulating therapies. J Neuroimmunol, 187:156-8.

Havrdova E, Giovannoni G, Hitchinson M, et al. 2007. Natalizumab increases the proportion of patients with multiple sclerosis who are disease free [abstract]. Mult Scler, 13(suppl 2):S170. Abstract P567.

IFNB Multiple Sclerosis Study Group. 1993. Interferon beta-1b is effective in relapsing-remitting multiple sclerosis. Clinical results of a multicenter, randomized, double-blind, placebo-controlled trial. Neurology, 43:655-61.

Jacobs LD, Cookfair DL, Rudick RA, et al. 1996. Intramuscular interferon beta-1a for disease progression in relapsing multiple sclerosis. Ann Neurol, 39:285-94.

Johnson KP, Brooks BR, Cohen JA, et al. 1995. Copolymer 1 reduces relapse rate and improves disability in relapsing-remitting multiple sclerosis: results of a phase III multicenter, double-blind, placebo-controlled trial. Neurology, 45:1268-76.

Kappos K, Bates D, Hartung H-P, et al. 2007. Natalizumab treatment for multiple sclerosis: recommendations for patient selection and monitoring. Lancet Neurol, 6:431-41.

Khatri B, Fox R, Koo A, et al. 2007. The effect of plasma exchange in accelerating clearance of natalizumab in patients with multiple sclerosis: results of the PLEX study [abstract]. Mult Scler, 13(Suppl 2): S172. Abstract P576.

Kleinschmidt-DeMasters BK, Tyler KL. 2005. Progressive multifocal leukoencephalopathy complicating treatment with natalizumab and interferon beta-1a for multiple sclerosis. $N$ Engl J Med, 353:369-74.

Langer-Gould A, Atlas SW, Bollen AW, et al. 2005. Progressive multifocal leukoencephalopathy in a patient treated with natalizumab. $N$ Engl $J$ Med, 353:375-81.

Lublin FD, Reingold SC. 1996. Defining the clinical course of multiple sclerosis: results of an international survey. Neurology, 46:907-11.

Major EO, Amemiya K, Tornatore CS, et al. 1992. Pathogenesis and molecular biology of progressive multifocal leukoencephalopathy, the JC virus-induced demyelinating disease of the human brain. Clin Microbiol Rev, 5:49-73.

McDonald WI, Compston A, Edan G, et al. 2001. Recommended diagnostic criteria for multiple sclerosis: guidelines from the International Panel in the Diagnosis of Multiple Sclerosis. Ann Neurol, 50:121-7.
Miller DH, Soon D, Fernando KT, et al. 2007. MRI outcomes in a placebo-controlled trial of natalizumab in relapsing MS. Neurology, 68:1390-401.

Noseworthy JH, Lucchinetti C, Rodriguez M, et al. 2000. Multiple sclerosis. N Engl J Med, 343:938-52.

O'Connor PW, Goodman A, Kappos L, et al. 2007 The efficacy of natalizumab monotherapy over 3 years of treatment in patients with relapsing multiple sclerosis. Neurology, 68(Suppl 1):A275. Abstract P06.082.

Panzara MA, Belcher G, Kooijmans M, et al. 2007. Use of natalizumab in patients with relapsing multiple sclerosis: updated safety results from TOUCH $^{\mathrm{TM}}$ and TYGRIS [abstract]. Mult Scler, 13(Suppl 2):S169. Abstract P565.

Phillips JT, Kappos L, O’Connor PW, et al. 2006. The effects of natalizumab monotherapy on multiple measures of disability progression in patients with multiple sclerosis. Presented at 58th Annual Meeting of the American Academy of Neurology; April 4; San Diego, CA.

Polman CH, O'Connor PW, Havrdova E, et al. 2006. A randomized, placebo-controlled trial of natalizumab for relapsing multiple sclerosis. N Engl J Med, 354:899-910.

PRISMS (Prevention of Relapses and Disability by Interferon beta-1a Subcutaneously in Multiple Sclerosis) Study Group. 1998. Randomised double-blind placebo-controlled study of interferon beta-1a in relapsing/ remitting multiple sclerosis. Lancet, 352:1498-504.

Rudick RA, Antel JA, Confavreux C, et al. 1997. Recommendations from the National Multiple Sclerosis Society Clinical Outcomes Assessment Task Force. Ann Neurol, 42:379-82.

Rudick RA, Fisher E, Lee J-C, et al. 1999. Use of brain parenchymal fraction to measure whole brain atrophy in relapsing-remitting MS. Neurology, 53:1698-704.

Rudick RA, Miller D, Hass S, et al. 2007. Health-related quality of life in multiple sclerosis: effects of natalizumab. Ann Neurol, 62:335-46.

Rudick RA, Stuart WH, Calabresi PA, et al. 2006. Natalizumab plus interferon beta-1 for relapsing multiple sclerosis. $N$ Engl $J$ Med, 354:911-23.

Schellekens H, Casadevall N. 2004. Immunogenicity of recombinant human proteins: causes and consequences. J Neurol, 251(Suppl 2): II/2-II/9.

Shitrit D, Lev N, Bar-Gil-Shitrit A, et al. 2005. Progressive multifocal leukoencephalopathy in transplant recipients. Transpl Int, 17:658-65.

Tysabri ${ }^{\circledR}$ [prescribing information]. 2007. Cambridge, MA: Biogen Idec, Inc.

US Food and Drug Administration, Center for Drug Evaluation and Research, Peripheral and Central Nervous System (PCNS) Advisory Committee. 2006. Briefing Document: Biogen Idec Biologics Marketing Application STN 125104/15, Natalizumab (Tysabri) for Multiple Sclerosis. Silver Spring, MD: US Department of Health and Human Services.

Van Assche G, Van Ranst M, Sciot R, et al. 2005. Progressive multifocal leukoencephalopathy after natalizumab therapy for Crohn's disease. N Engl J Med, 353:362-8.

Ware JE Jr, Sherbourne CD. 1992. The MOS 36-item short-form health survey (SF-36). I. Conceptual framework and item selection. Med Care, 30:473-483.

Yousry TA, Major EO, Ryschkewitsch C, et al. 2006. Evaluation of patients treated with natalizumab for progressive multifocal leukoencephalopathy. N Engl J Med, 354:924-33. 
